QUARTERLY OF APPLIED MATHEMATICS

VOLUME LXIV, NUMBER 2

JUNE 2006, PAGES 271-281

S $0033-569 \mathrm{X}(06) 00985-5$

Article electronically published on April 17, 2006

\title{
TOTAL GRAVITATIONAL ENERGY OF A SLIGHTLY ELLIPSOIDAL TRILAYER PLANET
}

\author{
BY \\ PAVEL GRINFELD (Department of Mathematics, Drexel University) \\ AND \\ JACK WISDOM (Department of EAPS, MIT)
}

\begin{abstract}
We use a perturbational technique to compute the total gravitational energy of a slightly ellipsoidal trilayer planet as a function of the two sets of Euler angles. A second order computation is required since the torque is proportional to the product of the ellipticities of the inner core and the mantle. Although we focus on ellipsoidal perturbations, the intermediate analytical expressions are valid for arbitrary small deformations of the spherical configuration.

The primary application of the expression for the total gravitational energy is in the Lagrangian formulation of dynamics. As a by-product, we determine the gravitationally stable equilibrium orientation of the rigid inner core. Due to symmetry, the six coaxial configurations are equilibrium. We show how to identify the stable configuration and to prove its uniqueness.
\end{abstract}

1. Introduction. The Earth and, quite likely, Mercury [3] are the solar system representatives of the class of planets that have a solid inner core, a fluid outer core and a solid mantle. For large planets these components are essentially spherical due to gravity, but not quite, and it is the deviations from the spherically symmetric configurations that are responsible for a number of interesting effects. A detailed description of a real planet's geometry is, of course, quite complicated - there are chemical inhomogeneities in the interior, uneven heating by the planet's star, and irregularities on the surface due to the elastic resistance of matter. We ignore these effects and employ an idealization that is designed to capture a specific small perturbation - the slight ellipticity of the planet. Ellipticity of planets is interesting because it causes precessions of the spin axis and librations which are deviations from uniform rotation. Ellipticity can be caused by the planet's rotation (the Earth) or heat convection (Mercury). While the Earth's ellipsoids are essentially axisymmetric, Mercury's famous resonant rotation [5] indicates that Mercury must be modeled as a triaxial ellipsoid.

Received May 10, 2005.

2000 Mathematics Subject Classification. Primary 81V17.

(C)2006 Brown University 
We consider a three-layer planet consisting of the rigid inner core $\Omega_{1}$, the fluid outer core $\Omega_{2}$ and the rigid mantle $\Omega_{3}$. Each layer is bounded by concentric triaxial ellipsoids of low eccentricity (of order of $\varepsilon$ ), and the boundaries of the mantle are coaxial. The respective densities are $\rho_{1}, \rho_{2}$, and $\rho_{3}$ (collectively, $\rho_{n}$ ). The configuration of the system can be specified by two sets of Euler angles: $\Theta_{1}, \Phi_{1}$ and $\Psi_{1}$ for the inner core and $\Theta_{3}$, $\Phi_{3}$ and $\Psi_{3}$ for the mantle.

We use a perturbational technique that utilizes the smallness of the parameter $\varepsilon$ to compute the total gravitational energy of the system as a function of Euler angles. A second order computation is required - Euler angles first appear in the terms of order $\varepsilon^{2}$ since the torque is proportional to the product of the ellipticities of the inner core and the mantle. Our technique is applied to arbitrary small deformations of the spherical configuration although in general it is applicable to arbitrary configurations.

The expression for the total gravitational energy finds its primary use in the Lagrangian formulation of the dynamic equations of motion. As a secondary application, we determine the stable equilibrium orientation of the rigid inner core. Due to symmetry, the six coaxial configurations are gravitationally equilibrium. At least one of them must be stable and it turns out that precisely one of them is. We show how to identify it and prove its uniqueness.

1.1. A note about the employed technique. Our technique, based on the calculus of moving surfaces, has many advantages. Our analytical expressions are short and have concise derivations. Our methods are applicable to arbitrary perturbations and generalize to any order in $\varepsilon$. Most importantly, the entire analysis is carried out by a formal variation of the total energy and does not require any geometric ingenuity or physical insight. (Some of the expressions below are large, but their complexity is due only to the coupled trilayer nature of the system.)

At the same time we realize that the calculus of moving surfaces is a substantial subject. To a reader not familiar with the technique, our approach may seem complicated. Even so, we believe that our conclusions, as well as many of our intermediate expressions, are easily interpretable without a thorough understanding of the calculus of moving surfaces, as long as the reader has an intuitive understanding of the interface velocity $C$. We do not include a description of the calculus of moving surfaces here, but it can be found in 2, including the definitions of all the quantities that we use and a summary of the key identities.

A more elementary derivation of the gravitational coupling in a triellipsoidal planet can be found in Szeto and $\mathrm{Xu}, 4$.

1.2. The evolution approach. We imagine a smooth evolution $\Omega(\tau)$ from the spherical configuration $\Omega$ to the nonspherical configuration $\Omega^{\prime}$. The evolution is indexed by a time-like parameter $\tau$ such that $\Omega(0)=\Omega$ and $\Omega(1)=\Omega^{\prime}$. We could have chosen $\tau$ to go from 0 to $\varepsilon$, but we prefer to imagine a slow evolution over a finite period of time rather than finite velocities over a short period.

The total energy of the system $E$ is also a smooth function of $\tau, E=E(\tau)$, and can be approximated by the first few (in our case, two) terms of the power series 
at time $\tau=1$ :

$$
E(1) \approx E(0)+\left.\frac{d E}{d \tau}\right|_{\tau=0}+\left.\frac{d^{2} E}{d \tau^{2}}\right|_{\tau=0}+O\left(\varepsilon^{3}\right) .
$$

Since the evolution is slow, the $n$-th derivative is $O\left(\varepsilon^{n}\right)$.

We mention two key identities from the calculus of moving surfaces pertaining to the time differentiation of volume and surface integrals:

$$
\begin{aligned}
\frac{d}{d \tau} \int_{\Omega} f(\tau, \Omega) d \Omega & =\int_{\Omega} \frac{\partial f(\tau, \Omega)}{\partial \tau} d \Omega+\int_{\partial \Omega} C f(\tau, S) d S \\
\frac{d}{d \tau} \int_{S} f(\tau, S) d S & =\int_{S} \frac{\delta f(t, S)}{\delta \tau} d S-\int_{S} C B_{\alpha}^{\alpha} f(\tau, S) d S
\end{aligned}
$$

In both identities $C$ is the invariant velocity of the surface referenced to the outward normal $\mathbf{N}$.

In the volume identity (2a),$\partial \Omega$ is the sufficiently smooth boundary of $\Omega, f(\tau, \Omega)$ indicates that $f$ is a field (vector or scalar) that depends on time and position, and $f(\tau, S)$ is the restriction of $f$ onto $\partial \Omega$. The identity can be interpreted as a moving surface generalization of the Fundamental Theorem of Calculus.

In the surface identity (2b),$S$ is a sufficiently smooth surface closed surface, and $f(\tau, S)$ indicates that $f$ is a field (also vector or scalar) that depends on time and surface position. $B_{\beta}^{\alpha}$ is the curvature tensor, and its trace $B_{\alpha}^{\alpha}$ is the mean curvature of the surface referenced to the outward normal. Finally, $\delta / \delta t$-derivative (although we have $\tau$ rather than the usual $t$ ) is the key operation in the calculus of moving surfaces which, for surface restrictions of spatial fields (such as the gravitational potential $\psi$ ), is defined analytically as

$$
\frac{\delta f}{\delta \tau}=\frac{\partial f(\tau, \Omega)}{\partial \tau}+C \mathbf{N} \cdot \nabla f
$$

The last identity is the moving surface equivalent of the classical Chain Rule.

Suppose that the field $f(\tau, \Omega)$ is discontinuous along an internal interface $S$ which is evolving with velocity $C$ and that $S$ is the only moving surface. Let $[f]_{S}$ denote the amount by which $f$ jumps across $S$, from the inside to the outside. Applying equation (2a) to both subdomains of $\Omega$, it is easy to obtain the following identity:

$$
\frac{d}{d \tau} \int_{\Omega} f(\tau, \Omega) d \Omega=\int_{\Omega} \frac{\partial f(\tau, \Omega)}{\partial \tau} d \Omega+\int_{S} C[f]_{S} d S .
$$

We frequently drop the subscript $S$ from $[f]_{S}$, letting $[f]$ represent the collection of jumps across the available interfaces, and use $[f]_{n}$ to indicate the jump in quantity $f$ across the $n$-th interface, $n=1,2,3$.

2. Energy variations. The total energy of the system is given as an integral over the interior of the planet

$$
E=\frac{1}{2} \int_{\Omega} d \Omega \rho \psi
$$

where the integration takes place cumulatively over $\Omega_{1}, \Omega_{2}$ and $\Omega_{3}, \rho$ is the mass density $\left(\rho_{1}, \rho_{2}\right.$, or $\rho_{3}$ depending on the domain) and $\psi$ is the gravitational potential expressed 
conceptually by

$$
\psi(\mathbf{z})=-G \int_{\Omega} \frac{\rho^{*}}{\left|\mathbf{z}-\mathbf{z}^{*}\right|} d \Omega^{*},
$$

where $G$ is the gravitational constant, $\mathbf{z}$ is the point at which $\psi$ is being evaluated, $\mathbf{z}^{*}$ varies of $\Omega$ and $\rho^{*}$ is shorthand for $\rho\left(\mathbf{z}^{*}\right)$.

2.1. The first variation. The first derivative of the energy $E$ with respect to the timelike parameter $\tau$ is obtained by applying identity (4) to the definition of energy (5):

$$
\frac{d E}{d \tau}=\frac{1}{2} \int_{\Omega} d \Omega \rho \frac{\partial \psi}{\partial \tau}+\frac{1}{2} \int_{\partial \Omega} d \Omega C[\rho] \psi .
$$

Next, substitute the definition of $\psi(6$ ) in the first term

$$
\frac{d E}{d \tau}=-\frac{1}{2} G \int_{\Omega} d \Omega \rho \frac{\partial}{\partial \tau} \int_{\Omega} \frac{\rho_{*}}{\left|\mathbf{z}-\mathbf{z}^{*}\right|} d \Omega^{*}+\frac{1}{2} \int_{\partial \Omega} d \Omega C[\rho] \psi,
$$

and apply (4) one more time

$$
\frac{d E}{d \tau}=-\frac{1}{2} G \int_{\Omega} d \Omega \rho \int_{\partial \Omega} C \frac{\left[\rho_{*}\right]}{\left|\mathbf{z}-\mathbf{z}^{*}\right|} d \Omega^{*}+\frac{1}{2} \int_{\partial \Omega} d \Omega C[\rho] \psi .
$$

In these identities, $\int_{\partial \Omega}$ is the sum of integrals over each of the three interfaces, and the discontinuity $[\rho]$ in density $\rho$ should be taken to be $[\rho]_{n}$, depending on which interface is being integrated. Similarly, $C$ represents the collection $C_{1}, C_{2}, C_{3}$.

We now exchange the order of integrations in the first integral - a delicate operation considering the singular nature of the integrand - and arrive at the final analytical expression for the first energy variation:

$$
\frac{d E}{d \tau}=\int_{\partial \Omega} d S C[\rho] \psi
$$

This expression applies to arbitrary initial geometries $\Omega$ and arbitrary perturbations $C$.

2.2. The second variation. The second variation of energy is obtained by differentiating equation (7) once more with respect to $\tau$. An application of (2b) yields

$$
\frac{d^{2} E}{d \tau^{2}}=\int_{S} d S[\rho] \frac{\delta(C \psi)}{\delta \tau}-\int_{S} d S[\rho] C^{2} B_{\alpha}^{\alpha} \psi,
$$

and since the $\delta / \delta t$-derivative satisfies the product rule, the first integral can be expanded as

$$
\frac{d^{2} E}{d \tau^{2}}=\int_{S} d S[\rho] \frac{\delta C}{\delta \tau} \psi+\int_{S} d S C[\rho] \frac{\delta \psi}{\delta \tau}-\int_{S} d S[\rho] C^{2} B_{\alpha}^{\alpha} \psi .
$$

The final expression is obtained by utilizing the chain rule (3) for $\delta \psi / \delta \tau$ :

$$
\frac{d^{2} E}{d \tau^{2}}=\int_{\partial \Omega} d S[\rho]\left(\frac{\delta C}{\delta \tau} \psi+C \frac{\partial \psi}{\partial \tau}+C \mathbf{N} \cdot \nabla \psi-C^{2} B_{\alpha}^{\alpha} \psi\right) .
$$

Much like the first variation, this expression applies to arbitrary initial geometries $\Omega$ and arbitrary perturbations $C$. 
2.3. Spherical configurations. From this point on, we restrict our attention to arbitrary perturbations of initially spherically symmetric geometries with radii $R_{1}, R_{2}$ and $R_{3}$. This assumption eliminates all but one term in equation (8).

Note that in the power series estimate (11), the derivatives are evaluated only at $\tau=0$. Therefore the integrations in equations (7) and (8) only need to be carried out over the unperturbed spherical domains, and only the initial values of $C, \psi$, and $B_{\alpha}^{\alpha}$ figure in the computation. Importantly, for spherically symmetric configurations, $\psi, \mathbf{N} \cdot \nabla \psi$ and $B_{\alpha}^{\alpha}$ are constant over each interface. We can ignore the terms with no functional dependence on the Euler angles, and that is every term except $C \partial \psi / \partial \tau$. We use a small circle over the equality sign $(\stackrel{\circ}{=})$ to indicate that terms independent of the Euler angles may have been dropped or added to the equation:

$$
E \stackrel{\circ}{=} \int_{\partial \Omega} d S C[\rho] \frac{\partial \psi}{\partial \tau} .
$$

The assumption of spherical symmetry allows us to represent each $C_{n}$ (at the initial moment $\tau=0)$ as a series in spherical harmonics $Y_{l m}(\theta, \phi)$

$$
C_{n}(\theta, \phi)=R_{n} C_{n}^{l m} Y_{l m}(\theta, \phi)
$$

with implied summation over $l$ and $m$. The factor of $R_{n}$ is introduced to nondimensionalize the constants $C_{n}^{l m}$. We take complex spherical harmonics $Y_{l m}(\theta, \phi)$ that are normalized to unity:

$$
\int_{|r|=1} Y_{l_{1} m_{1}}(\theta, \phi) Y_{l_{2} m_{2}}^{*}(\theta, \phi) d S=\delta_{l_{1} l_{2}} \delta_{m_{1} m_{2}} .
$$

We now turn our attention to $\partial \psi / \partial \tau$, the rate of change in the potential field $\psi$ induced by the simultaneous perturbations of the three boundaries. We employ a nondimensionalization scheme for $\psi$ and $\partial \psi / \partial t$ that introduces a length scale $R_{*}$ and a density $\rho_{*}$. We define the dimensionless densities $\varrho_{n}$ and dimensionless radii $Q_{n}$ :

$$
\varrho_{n}=\frac{\rho_{n}}{\rho_{*}}, \quad Q_{n}=\frac{R_{n}}{R_{*}},
$$

and a convenient quantity

$$
\Psi_{*}=\frac{4 \pi}{3} G \rho_{*} R_{*}^{2}
$$

that has dimensions of gravitational potential. $G$, again, is the gravitational constant.

The rate of change $\partial \psi / \partial \tau$ in the potential field $\psi$ at time $\tau=0$ can be expressed in terms of $C_{n}^{l m}$ as follows [2]:

$$
\frac{\partial \psi}{\partial \tau}(r, \theta, \phi)=\Psi_{*} \sum_{l, m}\left\{\begin{array}{rr}
A_{1}^{l m}\left(\frac{r}{R_{*}}\right)^{l}, & r \leq R_{1} \\
A_{2}^{l m}\left(\frac{r}{R_{*}}\right)^{l}+B_{2}^{l m}\left(\frac{r}{R_{*}}\right)^{-l-1}, & R_{1} \leq r \leq R_{2} \\
A_{3}^{l m}\left(\frac{r}{R_{*}}\right)^{l}+B_{3}^{l m}\left(\frac{r}{R_{*}}\right)^{-l-1}, & R_{2} \leq r \leq R_{3} \\
B_{4}^{l m}\left(\frac{r}{R_{*}}\right)^{-l-1}, & R_{3} \leq r
\end{array} \mid Y_{l m}(\theta, \phi),\right.
$$


where $A_{n}^{l m}$ and $B_{n}^{l m}$ are linear combinations of $C_{n}^{l m}$ :

$$
\left[\begin{array}{c}
A_{1}^{l m} \\
A_{2}^{l m} \\
A_{3}^{l m} \\
B_{2}^{l m} \\
B_{3}^{l m} \\
B_{4}^{l m}
\end{array}\right]=-\frac{3}{2 l+1}\left[\begin{array}{ccc}
Q_{1}^{-l+2} & Q_{2}^{-l+2} & Q_{3}^{-l+2} \\
0 & Q_{2}^{-l+2} & Q_{3}^{-l+2} \\
0 & 0 & Q_{3}^{-l+2} \\
Q_{1}^{l+3} & 0 & 0 \\
Q_{1}^{l+3} & Q_{2}^{l+3} & 0 \\
Q_{1}^{l+3} & Q_{2}^{l+3} & Q_{3}^{l+3}
\end{array}\right]\left[\begin{array}{l}
{[\varrho]_{1} C_{1}^{l m}} \\
{[\varrho]_{2} C_{2}^{l m}} \\
{[\varrho]_{3} C_{3}^{l m}}
\end{array}\right]
$$

Of particular interest is the value of $\partial \psi / \partial \tau$ at the interfaces $S_{1}, S_{2}$, and $S_{3}$ :

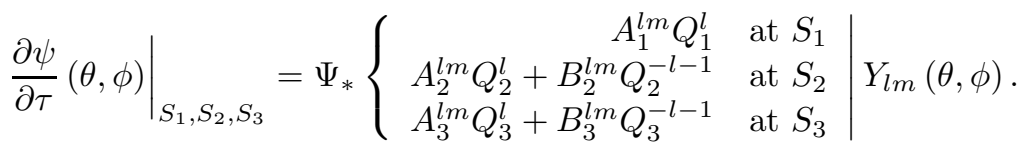

We now have all of the ingredients necessary to express the orientation-dependent contribution to energy (9) as a quadratic form in $C_{n}^{l m}$. We first expand the integral over the three interfaces and take a formal complex conjugate of $C$ in order to take advantage of our choice of spherical harmonics (11):

$$
E \stackrel{\circ}{=} \int_{S_{1}} d S C_{1}^{*}[\rho]_{1}\left(\frac{\partial \psi}{\partial \tau}\right)_{S_{1}}+\int_{S_{2}} d S C_{2}^{*}[\rho]_{2}\left(\frac{\partial \psi}{\partial \tau}\right)_{S_{2}}+\int_{S_{3}} d S C_{3}^{*}[\rho]_{3}\left(\frac{\partial \psi}{\partial \tau}\right)_{S_{3}} .
$$

Due to the orthonormality of the spherical harmonics over the unit sphere (thus the additional factors of $R_{n}^{2}$ ), each integral becomes a sum

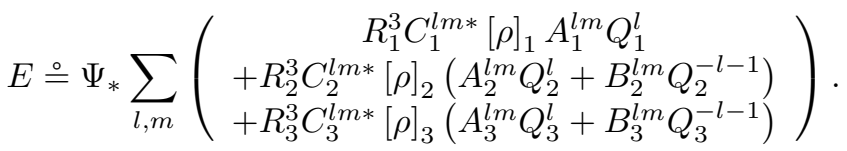

We nondimensionalize the above expression by factoring out $\rho_{*} R_{*}^{3}$ :

$$
E \doteq \Psi_{*} \rho_{*} R_{*}^{3} \sum_{l, m}\left(\begin{array}{c}
C_{1}^{l m *}[\varrho]_{1} A_{1}^{l m} Q_{1}^{l+3} \\
+C_{2}^{l m *}[\varrho]_{2}\left(A_{2}^{l m} Q_{2}^{l+3}+B_{2}^{l m} Q_{2}^{-l+2}\right) \\
+C_{3}^{l m *}[\varrho]_{3}\left(A_{3}^{l m} Q_{3}^{l+3}+B_{3}^{l m} Q_{3}^{-l+2}\right)
\end{array}\right),
$$

or, in matrix form,

$$
\begin{aligned}
& E \triangleq \Psi_{*} \rho_{*} R_{*}^{3} \sum_{l, m}\left[\begin{array}{l}
C_{1}^{l m} \\
C_{2}^{l m} \\
C_{3}^{l m}
\end{array}\right]^{*}\left[\begin{array}{ccccc}
{[\varrho]_{1} Q_{1}^{l+3}} & 0 & 0 & 0 & 0 \\
0 & {[\varrho]_{2} Q_{2}^{l+3}} & 0 & {[\varrho]_{2} Q_{2}^{-l+2}} & 0 \\
0 & 0 & {[\varrho]_{3} Q_{3}^{l+3}} & 0 & {[\varrho]_{3} Q_{3}^{-l+2}}
\end{array}\right] \\
& \times\left[\begin{array}{c}
A_{1}^{l m} \\
A_{2}^{l m} \\
A_{3}^{l m} \\
B_{2}^{l m} \\
B_{3}^{l m}
\end{array}\right] .
\end{aligned}
$$


Substituting the expressions for $A_{n}^{l m}$ and $B_{n}^{l m}$ in terms of $C_{n}^{l m}$, equation (14), we obtain the final expression for the angle dependent contribution to energy:

$$
\begin{aligned}
& E \stackrel{\circ}{=} \Psi_{*} \varrho_{*} R_{*}^{3} \sum_{l, m} \frac{3}{2 l+1} \\
& \times\left[\begin{array}{l}
C_{1}^{l m} \\
C_{2}^{l m} \\
C_{3}^{l m}
\end{array}\right]^{*}\left[\begin{array}{ccc}
{[\varrho]_{1}^{2} Q_{1}^{5}} & {[\varrho]_{1}[\varrho]_{2} Q_{1}^{l+3} Q_{2}^{-l+2}} & {[\varrho]_{1}[\varrho]_{3} Q_{1}^{l+3} Q_{3}^{-l+2}} \\
{[\varrho]_{2}[\varrho]_{1} Q_{2}^{-l+2} Q_{1}^{l+3}} & {[\varrho]_{2}^{2} Q_{2}^{5}} & {[\varrho]_{2}[\varrho]_{3} Q_{2}^{l+3} Q_{3}^{-l+2}} \\
{[\varrho]_{3}[\varrho]_{1} Q_{3}^{-l+2} Q_{1}^{l+3}} & {[\varrho]_{3}[\varrho]_{2} Q_{3}^{-l+2} Q_{2}^{l+3}} & {[\varrho]_{3}^{2} Q_{3}^{5}}
\end{array}\right]\left[\begin{array}{c}
C_{1}^{l m} \\
C_{2}^{l m} \\
C_{3}^{l m}
\end{array}\right]
\end{aligned}
$$

These matrix products must be interpreted carefully since $C_{n}^{l m}$ are vectors of length $2 l+1$. A less controversial way to write the above expression is

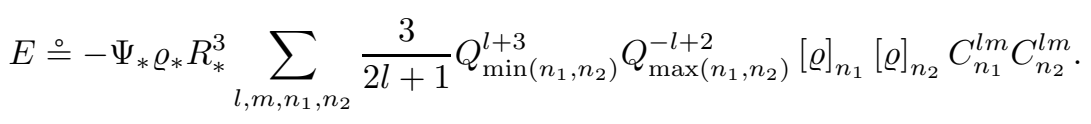

We now turn to the task of computing $C_{n}^{l m}$ for our specific perturbation.

3. Computing $E$ for rotations in the horizontal plane. In this section we focus on configurations for which the inner core and the mantle rotate about the $z$-axis and only $\Phi_{1}$ and $\Phi_{2}$ are nonzero. The case of arbitrary orientations is considered in the next section, but this case is sufficient to identify the stable orientation of the inner core.

Suppose that we have a near-spherical ellipsoid with semiaxes $R(1+\varepsilon a), R(1+\varepsilon b)$, $R(1+\varepsilon c)$. Then an appropriate evolution of the semiaxes is $R(1+\varepsilon \tau a), R(1+\varepsilon \tau b)$, $R(1+\varepsilon \tau c)$, which leads us to the following estimate of $C_{\text {Generic }}$ at $\tau=0$ in polar coordinates on the sphere when the semiaxes are aligned with corresponding Cartesian coordinate axes (thus "Generic"):

$$
\left.C_{\text {Generic }}\right|_{\tau=0}=\varepsilon R\left(a \sin ^{2} \theta \cos ^{2} \phi+b \sin ^{2} \theta \sin ^{2} \phi+c \cos ^{2} \theta\right) .
$$

The decomposition of $C_{\text {Generic }}$ with respect to spherical harmonics results in only two sets of nonzero coefficients corresponding to $l=0$ and $l=2$ :

$$
\begin{aligned}
& C_{\text {Generic }}^{00}= \varepsilon \sqrt{\frac{4 \pi}{9}}(a+b+c), \\
& C_{\text {Generic }}^{2 m}=\varepsilon\left[\begin{array}{c}
\sqrt{\frac{2 \pi}{15}}(a-b) \\
0 \\
\sqrt{\frac{4 \pi}{45}}(2 c-a-b) \\
0 \\
\sqrt{\frac{2 \pi}{15}}(a-b)
\end{array}\right] .
\end{aligned}
$$

If the ellipsoid is rotated counterclockwise about the $z$-axis by an angle $\Phi$, then to obtain its decomposition, we first represent it as a linear combination of "intermediate" likewise rotated harmonics - and, of course, get the same values as (19a) and (19b) - and then express the rotated harmonics with respect to the standard harmonics. This simple 
procedure yields the following coefficients $C^{l m}$ :

$$
\begin{aligned}
& C^{00}= \varepsilon \sqrt{\frac{4 \pi}{9}}(a+b+c), \Phi \neq 0, \\
& C^{2 m}=\varepsilon\left[\begin{array}{c}
\sqrt{\frac{2 \pi}{15}}(a-b) e^{-2 i \Phi} \\
0 \\
\sqrt{\frac{4 \pi}{45}}(2 c-a-b) \\
0 \\
\sqrt{\frac{2 \pi}{15}}(a-b) e^{2 i \Phi}
\end{array}\right], \Phi \neq 0 .
\end{aligned}
$$

The idea of rotating harmonics to follow the rotation of the ellipsoid carries over to the case of arbitrary orientation.

Let the three sets of coefficients obtained for the three interfaces of the planet be $C_{1}^{l m}$, $C_{2}^{l m}$, and $C_{3}^{l m}$. The coefficients $C_{1}^{00}, C_{2}^{00}$, and $C_{3}^{00}$ corresponding to $l=0, m=0$ have no angular dependence. Thus only $l=2$ matters, and equation (17) simplifies to

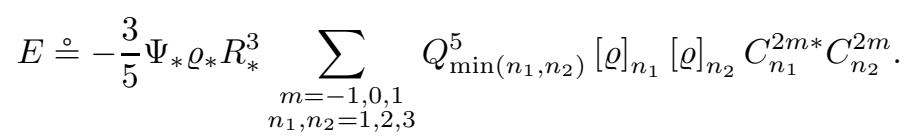

The nonzero contributions come only from the $\left(n_{1}, n_{2}\right)$ pairs $(1,2),(2,1),(1,3)$, and $(3,1)$, and because the quadratic form is symmetric and the product $C_{n_{1}}^{2 m *} C_{n_{2}}^{2 m}$ is real, we get

$$
E \stackrel{\circ}{=} \frac{6}{5} \Psi_{*} \varrho_{*}[\varrho]_{1} R_{*}^{3} Q_{1}^{5} \sum_{m}\left([\varrho]_{2} C_{1}^{2 m *} C_{2}^{2 m}+[\varrho]_{3} C_{1}^{2 m *} C_{3}^{2 m}\right) .
$$

Substituting coefficients (20a) and (20b), we obtain the nondimensionalized estimate

$$
E \stackrel{\varrho}{=} \frac{6}{5} \frac{2 \pi}{15} \Psi_{*} \varrho_{*}[\varrho]_{1} R_{*}^{3} Q_{1}^{5}\left(a_{1}-b_{1}\right)\left([\varrho]_{2}\left(a_{2}-b_{2}\right)+[\varrho]_{3}\left(a_{3}-b_{3}\right)\right) \cos 2\left(\Phi_{1}-\Phi_{2}\right) .
$$

Recalling the definition of $\Psi_{*}$ we present the final expression for the orientation dependent contribution to energy:

$$
E \stackrel{\circ}{=} \frac{32}{75} \pi^{2} G R_{1}^{5}[\rho]_{1}\left(a_{1}-b_{1}\right)\left([\rho]_{2}\left(a_{2}-b_{2}\right)+[\rho]_{3}\left(a_{3}-b_{3}\right)\right) \sin ^{2}\left(\Phi_{1}-\Phi_{2}\right) .
$$

3.1. Stability analysis. The system is stable with respect to rotations about the $z$-axis if the expression

$$
[\rho]_{1}\left(a_{1}-b_{1}\right)\left([\rho]_{2}\left(a_{2}-b_{2}\right)+[\rho]_{3}\left(a_{3}-b_{3}\right)\right)
$$

is positive. Similar arguments for the $x$-axis and the $y$-axis show that the system is stable in the overall sense if all of the following inequalities hold:

$$
\begin{array}{r}
{[\rho]_{1}\left(a_{1}-b_{1}\right)\left([\rho]_{2}\left(a_{2}-b_{2}\right)+[\rho]_{3}\left(a_{3}-b_{3}\right)\right)>0,} \\
{[\rho]_{1}\left(b_{1}-c_{1}\right)\left([\rho]_{2}\left(b_{2}-c_{2}\right)+[\rho]_{3}\left(b_{3}-c_{3}\right)\right)>0,} \\
{[\rho]_{1}\left(c_{1}-a_{1}\right)\left([\rho]_{2}\left(c_{2}-a_{2}\right)+[\rho]_{3}\left(c_{3}-a_{3}\right)\right)>0 .}
\end{array}
$$


These expressions make it very easy to judge stability for a given orientation and to show that only a single coaxial configuration is stable. Note that the three quantities

$$
\begin{aligned}
& {[\rho]_{2}\left(a_{2}-b_{2}\right)+[\rho]_{3}\left(a_{3}-b_{3}\right),} \\
& {[\rho]_{2}\left(b_{2}-c_{2}\right)+[\rho]_{3}\left(b_{3}-c_{3}\right),} \\
& {[\rho]_{2}\left(c_{2}-a_{2}\right)+[\rho]_{3}\left(c_{3}-a_{3}\right),}
\end{aligned}
$$

add up to zero. Therefore, two of them have one sign, and the third has the other. For example, assume that the first two quantities are positive. Then equations (22a)-(22c) indicate that the stable orientation is such that $a_{1}>b_{1}>c_{1}$.

In particular, if we consider a typical planet such that $\rho_{1}>\rho_{2}>\rho_{3}, a_{2}>b_{2}>c_{2}$ and $a_{3}>b_{3}>c_{3}$, then the combination of signs just considered applies, and we conclude that a stable orientation of the inner core is one for which $a_{1}>b_{1}>c_{1}$. In other words, the $i$-th longest semiaxis of the inner core must be aligned with the $i$-th longest semiaxis of the mantle.

4. Computing $E$ for arbitrary orientations. We now consider a general orientation of a triaxial ellipsoid given by the Euler angles $\Theta, \Phi$, and $\Psi$. We use the same approach as we did for rotations in the horizontal plane. That is, we decompose the deformation with respect to appropriately rotated harmonics $Y_{l m}^{\prime}$ (and obtain precisely the same expressions (19a) and (19b) $)$, and then express the rotated harmonics $Y_{l m}^{\prime}$ with respect to the standard harmonics $Y_{l m}$. The second step is accomplished by a consecutive application of three rotation matrices that correspond to the three Euler rotations. If $Y_{2 m}^{\prime}$ are obtained by rotating $Y_{2 m}$ by Euler angles $\Theta, \Phi$, and $\Psi$, then

$$
Y_{2 m}^{\prime}(\theta, \phi)=H_{Z}(\Phi) H_{Y}(\Theta) H_{Z}(\Psi) Y_{2 m}(\theta, \phi),
$$

and therefore

$$
C_{n}^{2 m}=H_{Z}(\Phi) H_{Y}\left(\Theta_{n}\right) H_{Z}\left(\Psi_{n}\right) C_{n, \text { Generic }}^{2 m},
$$

where $\Theta_{1}, \Phi_{1}, \Psi_{1}$ are taken for $n=1$, and $\Theta_{3}, \Phi_{3}, \Psi_{3}$ are taken for $n=2,3$, and

$$
H_{Z}(\Psi)=\left[\begin{array}{ccccc}
e^{-2 i \Psi} & 0 & 0 & 0 & 0 \\
0 & e^{-i \Psi} & 0 & 0 & 0 \\
0 & 0 & 1 & 0 & 0 \\
0 & 0 & 0 & e^{i \Psi} & 0 \\
0 & 0 & 0 & 0 & e^{2 i \Psi}
\end{array}\right]
$$

and

$$
H_{Y}(\Theta)=\frac{1}{4}\left[\begin{array}{cccc}
(\cos \Theta+1)^{2} & 2(\cos \Theta+1) \sin \Theta & \sqrt{6} \sin ^{2} \Theta & \rightarrow \\
-2(\cos \Theta+1) \sin \Theta & 2(\cos \Theta+1)(2 \cos \Theta-1) & 2 \sqrt{6} \sin \Theta \cos \Theta & \rightarrow \\
\sqrt{6} \sin ^{2} \Theta & -2 \sqrt{6} \sin \Theta \cos \Theta & -2+6 \cos ^{2} \Theta & \rightarrow \\
2(\cos \Theta-1) \sin \Theta & -2(\cos \Theta-1)(2 \cos \Theta+1) & -2 \sqrt{6} \sin ^{2} \cos \Theta & \rightarrow \\
(\cos \Theta-1)^{2} & 2(\cos \Theta-1) \sin \Theta & \sqrt{6} \sin ^{2} \Theta & \rightarrow \\
\rightarrow & -2(\cos \Theta-1) \sin \Theta & (\cos \Theta-1)^{2} & \\
\rightarrow & -2(2 \cos \Theta+1)(\cos \Theta-1) & -2(\cos \Theta-1) \sin \Theta \\
\rightarrow & 2 \sqrt{6} \sin \Theta \cos \Theta & \sqrt{6} \sin ^{2} \Theta \\
\rightarrow & 2(\cos \Theta+1)(2 \cos \Theta-1) & 2(\cos \Theta+1) \sin \Theta & \\
\rightarrow & -2(\cos \Theta+1) \sin \Theta & (\cos \Theta+1)^{2}
\end{array}\right] .
$$


Our analysis is now complete. Given the orientations of the inner core and the mantle expressed in Euler angles, the perturbation expansion coefficients can be determined by equation (24), where $\Theta_{1}, \Phi_{1}$, and $\Psi_{1}$ are used to evaluate $C_{1}^{l m}$, and $\Theta_{3}, \Phi_{3}$, and $\Psi_{3}$ are used to evaluate $C_{2}^{l m}$ and $C_{3}^{l m}$. Next, the energy perturbation can be computed by equation (17) for arbitrary perturbations and by equation (21) for ellipsoidal perturbations.

4.1. Conclusions. We have applied the method of moving surfaces to compute the second order perturbation to the total gravitational energy of a slightly nonspherical triaxial planet. The presented technique applies to arbitrary perturbations of the spherical configuration. As an illustration we considered ellipsoidal perturbations that find frequent applications in planetary dynamics.

The derived expressions can be employed in the Lagrangian formulation of dynamics. The resulting expressions are quite complex, and it would be a challenging task to derive the Lagrangian equations of motion analytically. It turns out that for ellipsoidal perturbations, the Newtonian approach is simpler since the coupling torque can be computed according to Szeto and $\mathrm{Xu}$, 4], assuming no outer core. When the outer core is accounted for, Szeto and Xu's approach becomes quite complicated as well. Finally, if nonellipsoidal perturbations are considered, the use of a general morphological technique such as ours is unavoidable.

5. Appendix: Moment of inertia perturbation. We have provided an estimate for the potential energy accumulated between the inner core and the mantle. The Lagrangian approach also requires an expression for the kinetic energy of the system. We would therefore like to present a computation for the first order correction to the principal moments of inertia of a rigid body of constant density. The total kinetic energy of the rigid body can be expressed in terms of the tensor of inertia, Euler angles $\Theta$, $\Phi$, and $\Psi$, and their time derivatives $\dot{\Theta}, \dot{\Phi}$, and $\dot{\Psi}$.

The moments of inertia with respect to the $x$-, $y$-, and $z$-axes, usually denoted by $A$, $B$, and $C$, are defined as

$$
\begin{aligned}
A & =\rho \int_{\Omega} d \Omega\left(y^{2}+z^{2}\right), \\
B & =\rho \int_{\Omega} d \Omega\left(z^{2}+x^{2}\right), \\
C & =\rho \int_{\Omega} d \Omega\left(x^{2}+y^{2}\right) .
\end{aligned}
$$

The first perturbation of $C$ is obtained by applying identity (2a):

$$
\frac{d C}{d \tau}=\rho \int_{S} d S C_{\text {as in 19a)! }}\left(x^{2}+y^{2}\right),
$$

which, for an ellipsoidal perturbation (18), yields

$$
\begin{aligned}
\frac{d C}{d \tau} & =\rho \int_{-\pi}^{\pi} \int_{0}^{\pi} \varepsilon R\left(a \sin ^{2} \theta \cos ^{2} \phi+b \sin ^{2} \theta \sin ^{2} \phi+c \cos ^{2} \theta\right)\left(R^{2} \sin ^{2} \theta\right) R^{2} \sin \theta d \theta d \phi \\
& =\frac{8}{15} \pi \rho R^{5} \varepsilon(2 a+2 b+c)
\end{aligned}
$$


For the unperturbed sphere $C=\frac{8}{15} \pi \rho R^{5}$, we have

$$
\frac{d C}{d \tau}=\varepsilon(2 a+2 b+c) C .
$$

Therefore, the out-of-roundness parameter $(B-A) / C$ is

$$
\begin{aligned}
\frac{B-A}{C} & =\frac{\left(C+\frac{d B}{d \tau}+O\left(\varepsilon^{2}\right)\right)-\left(C+\frac{d A}{d \tau}+O\left(\varepsilon^{2}\right)\right)}{C+\frac{d C}{d \tau}+O\left(\varepsilon^{2}\right)} \\
& =\varepsilon(2 a+b+2 c)-\varepsilon(a+2 b+2 c)+O\left(\varepsilon^{2}\right) \\
& =\varepsilon(a-b)+O\left(\varepsilon^{2}\right) .
\end{aligned}
$$

Thus, to first order

$$
\frac{B-A}{C}=\varepsilon(a-b)
$$

\section{REFERENCES}

[1] Grinfeld P., 2003. Boundary Perturbations of Laplace Eigenvalues. Applications to Polygons and Electron Bubbles. Thesis, Department of Mathematics, MIT.

[2] Grinfeld P., Wisdom J. (Submitted). A way to compute the gravitational potential for near-spherical geometries.

[3] G. Schubert, M.N. Ross, D.J. Stevenson, and T. Spohn, 1988. Mercury's Thermal History and the Generation of Its Magnetic Field. Mercury (Univ. of Ariz Press), 429-460.

[4] A.M.K. Szeto and S. Xu (1997). Gravitational Coupling in a Triaxial Ellipsoidal Earth, J. Geophys. Res., vol. 102, 27651-27657.

[5] G.J. Sussman and J. Wisdom. Structure and Interpretation of Classical Mechanics, MIT Press, 2001. MR1825485 (2002e:70001) 